\title{
Structural Analysis and Five-Layered Control Frame Work for Underground Pipe Inspection Robot
}

\author{
Ajin M, Satheeshkumargopal \\ Department of Mechanical Engineering, SSN College of Engineering, India
}

\begin{tabular}{l}
\hline \hline Article Info \\
\hline Article history: \\
Received Sep 20, 2016 \\
Revised Nov 12, 2016 \\
Accepted Nov 25, 2016 \\
\hline
\end{tabular}

\section{Keyword:}

Control architecture

Fuzzy based output

Non-dimensional parameter

Pipeline robot

Structural analysis

\begin{abstract}
Design of a robot generally involves various types of analyses that invariably include structural, numerical, kinematic, dynamic and experimental analysis. A robot designed as a solution for social problems would have to undergo all these stages before it is incorporated in-situ. The authors in continuation to their earlier work [1] are presenting the results of structural analysis in this paper. Initially a detailed description of the anatomy of the proposed robot is provided for clarity along with the identified design parameters. The primary design parameter 'load to power ratio' establishes certain geometric and structural constrains on the design of links comprising the robot. This relationship is studied in order to improve to the load to power ratio of the robot. A significant reduction in weight is achieved by working on these variables which leads to the improvement in the functional aspects too, viz., reduced torque requirement from the motor. Upon finalizing on the choice of motors and sensors the 5 layered control architecture based on statespace representation is also presented. Fuzzy logic based control algorithm is proposed to unite the layered approach depending on the task allocated to the robot.
\end{abstract}

Copyright $\odot 2016$ Institute of Advanced Engineering and Science. All rights reserved.

\section{Corresponding Author:}

Ajin M,

Departement of Mechanical Engineering,

SSN College of Engineering,

Kalavakkam, India - 603110

Email: ajin.ganesh@gmail.com

\section{INTRODUCTION}

Modern day researchers, in more numbers than ever, strive to develop solutions for problems that seem to alleviate the social problems faced these days. One such solution is in-pipe mechanism for inspection tasks inside pipelines, where the robot is desired to enter and clear narrow and complex pipelines. In this paper a unique reconfigurable robot which would be used for inspection of sewage pipeline is proposed. Some of the tasks preformed by an in-pipe robot include Visual Testing and Non-Destructive Testing (NDT) [2]. This paper introduced adaptability of the robots for varying diameters, though they face problems related to the feeding air tube and the robot is for smaller diameter pipes. The need for providing a low power to weight (or load) ratio was discussed by the authors.

A thorough study of robots through structural analysis would reveal the strength and stiffness of the proposed design, even in the case of dragon fly wing [3]. Horodinca et al., [4] presented a simple architecture for in-pipe inspection robots for different pipe diameters but their robots were devoid of any reconfigurability. Different robots were made for different pipe diameters. And also though the design of the robots was unique they could handle only a payload of 5 N. Similarly stress analysis reveals the load bearing capacity of the robot even in pipeline applications [5].

Though there are methods for generation of kinematic chains with a given number of links and degree of freedom through structural synthesis [6], development of model and performance analysis is still 
done from the perspective of control system design even in the perspective of wheel skidding and slipping [7]. Though the kinematic analysis was presented in the earlier paper [1] singularity analysis as performed by Zlatanov et. al., [8] is not needed since within the work envelope the external forces would nullify the outcome and would be treated as a nonfunctional effect.

In this paper the anatomy along with the parameters for characterizations of the robot is discussed initially. It is then followed by a discussion on structural analysis and the effect of non dimensional parameters. Finally the proposed control architecture is united through Fuzzy logic algorithm.

\section{ANATOMY OF ROBOT}

\subsection{Description of the robot}

The proposed robot is designed to operate in the closed pipe line of the underground drainage system for the inspection of clog inside the pipeline. The robot is capable of moving in variable diameter pipelines ranging from $200 \mathrm{~mm}$ to $350 \mathrm{~mm}$ with the help of lead screw mechanism. This reconfigurable capability reduces the efforts in making individual robots for individual pipe sizes. The robot has four links at the front and four links at the back, comprising the legs. This provides stability to the robot in static conditions by allowing the CG to fall within the support polygon. The control design ensures to validate the presence of $\mathrm{CG}$ within the support polygon with relevant inputs from the sensors, even in fully extended condition. Fig. 1. shows the model of the proposed in-pipe inspection robot. All the joints in this robot are active joints and hence transmit torque.

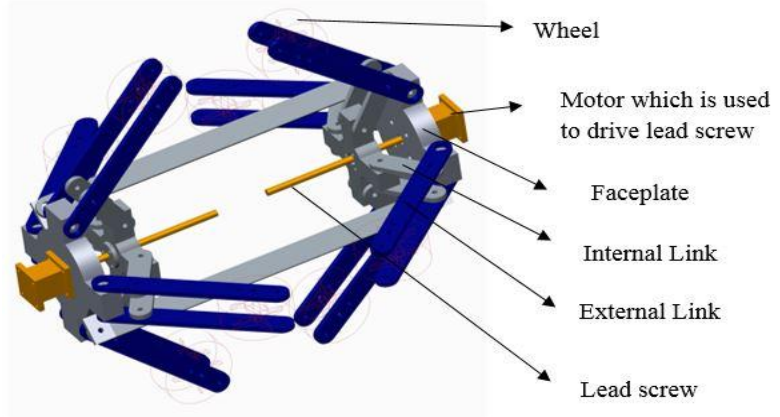

Figure 1. CAD model of proposed robot

\subsection{Design aspects of the proposed robot}

Structural analysis of a robot reveals the optimized geometry, material of the parts and torque requirements of the motors, among others. To perform the analysis, the parameters identified for designing the robot to improve performance and to meet reconfigurability are listed below:

- Robot weight, Rw

- Robot payload, Rp

- Load to Weight ratio, $\gamma$

- Length of the robot, LR

- Spanning diameter, DR

- Length to Span ratio, $\mu$

- Torque requirement, TR

- Tractive effort, TE

- Speed of the robot and

- Connectivity of the robot

The design parameters based on geometry of the robot parts are discussed in this paper. The discussion on reconfigurability and the associated parameters [9] is reserved as it is not in the scope of this paper. 


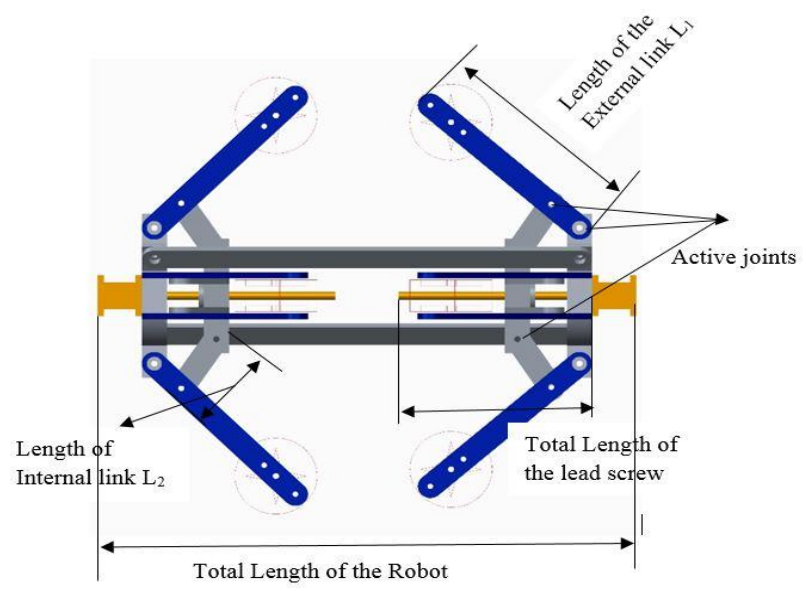

Figure 2. Parameters considered while designing the robot

The major parameters listed above are dependent on atleast two or more sub parameters. For ex., the length of the robot is dependent on the sub parameters like lead screw length, the distance between the first and second lead screw and face plates thickness. Figure 2 shows some of the important sub-parameters. The other sub-parameters are listed below:

- external arm length

- internal arm length

- arm Thickness

- wheel diameter

- face plate diameter

- small face plate diameter

- external arm rotation angles

- flange height at above the face plate

- flange height at above the small face plate

\section{STRUCTURAL ANALYSIS OF THE ROBOT}

Using the scientific procedure of structural analysis, the reaction of a robot body to definite loads is determined. This response is found out by finding the internal stress, displacement and deformation. The whole robot body including arm, faceplate is made up of Aluminum 606. The density of that 6061 alloy is $0.02647755 \mathrm{~N} / \mathrm{mm} 3$ and its Young's modulus is $70000 \mathrm{~N} / \mathrm{mm} 2(70 \mathrm{GPa})$. Three different thicknesses are chosen to vary the robot weight $\&$ payload and the tradeoff is studied to optimize these parameters for better torque requirement.

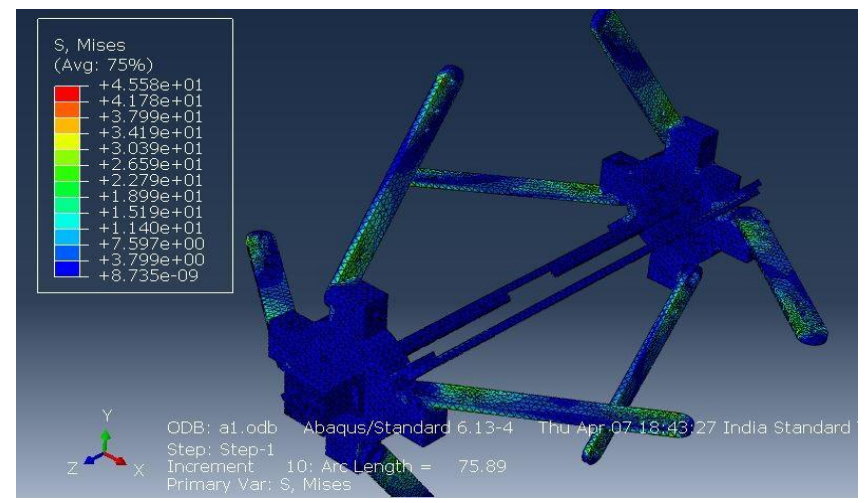

Figure 3. Stress distribution with thickness of arm is $5 \mathrm{~mm}$ 


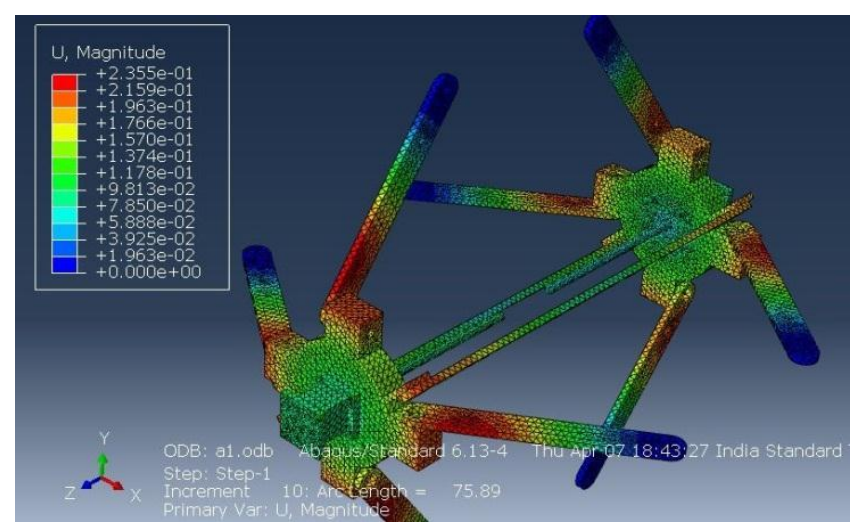

Figure 4. Displacement when thickness of arm is $5 \mathrm{~mm}$

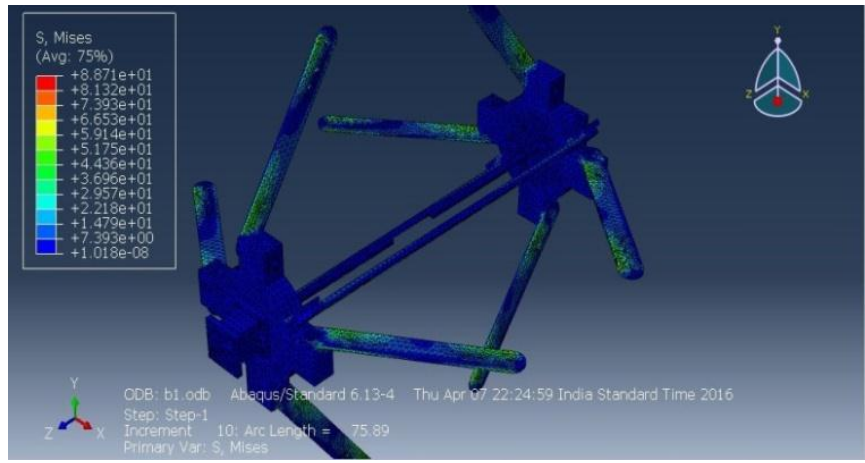

Figure 5. Stress distribution with thickness of arm is $3 \mathrm{~mm}$

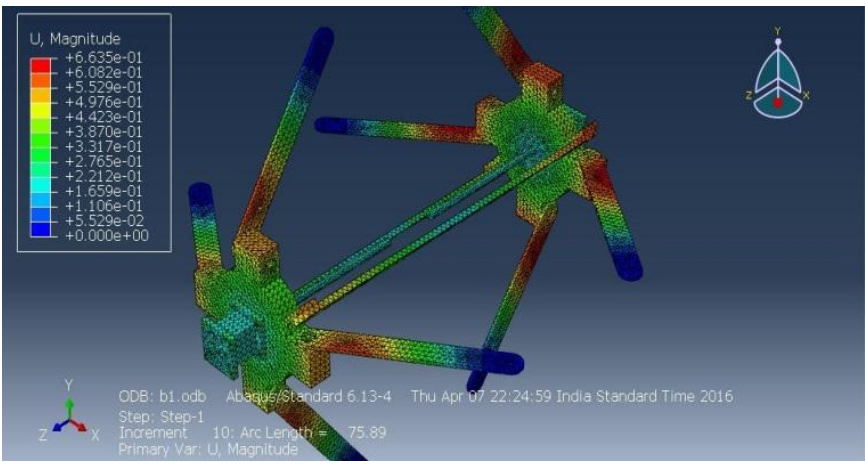

Figure 6. Displacement when thickness of arm is $3 \mathrm{~mm}$

Figure $3 \& 4$ show the output of the structural analysis of different robot link thickness of $5 \mathrm{~mm}$. The pressure generally needed to remove the obstruction inside the pipe is 0.002 to $0.006 \mathrm{~N} / \mathrm{mm} 2$. Under normal working condition in flowing water, it is assumed that the pressure acting on the robot is half of the minimum pressure required to remove the clog, i.e. $0.001 \mathrm{~N} / \mathrm{mm} 2(1000 \mathrm{~Pa})$, inside the sewer pipelines. This is the load applied on the robot for which the structural analysis is performed. All the legs are assumed to be in contact with the pipes and the traction force is assumed to be equal in all legs. Figure. $5 \& 6$ and $7 \& 8$ show the output for robot link thicknesses of $3 \mathrm{~mm}$ and $1 \mathrm{~mm}$. Though the displacement is less for $5 \mathrm{~mm}$ plate, the weight of the parts increases and hence the tradeoff.

\subsection{Result and discussion}

Figure 9 shows the result taken from a selected nodal point which displays the stress distribution and also the displacement for 10 increments for different arm thickness such as $1 \mathrm{~mm}, 3 \mathrm{~mm}$ and $5 \mathrm{~mm}$ under 
applied load. The maximum nodal displacement for the arm thickness $1 \mathrm{~mm}, 3 \mathrm{~mm}$ and $5 \mathrm{~mm}$ are as 1.63 $\mathrm{mm}, 0.57 \mathrm{~mm}$ and $0.22 \mathrm{~mm}$ respectively. The conclusion is that the structure won't have any effect for the applied load and hence the thickness of $3 \mathrm{~mm}$ can be used for the robot arm.

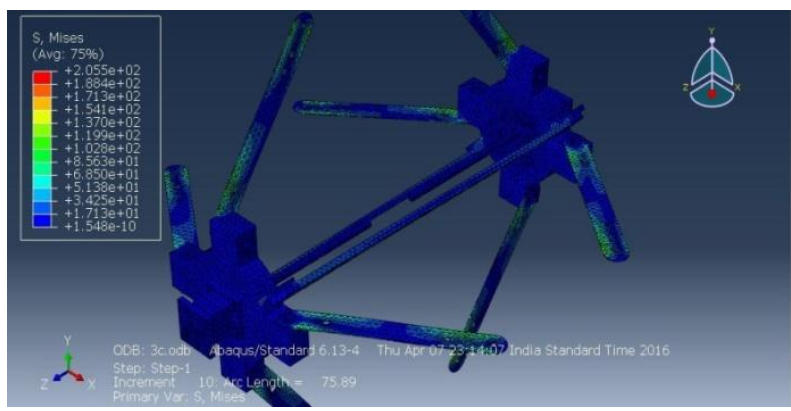

Figure 7. Stress distribution with thickness of arm is $1 \mathrm{~mm}$

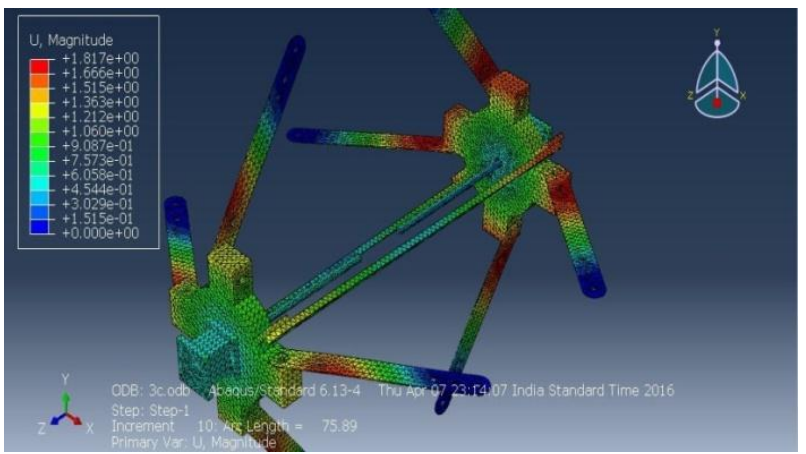

Figure 8. Displacement when thickness of arm is $1 \mathrm{~mm}$

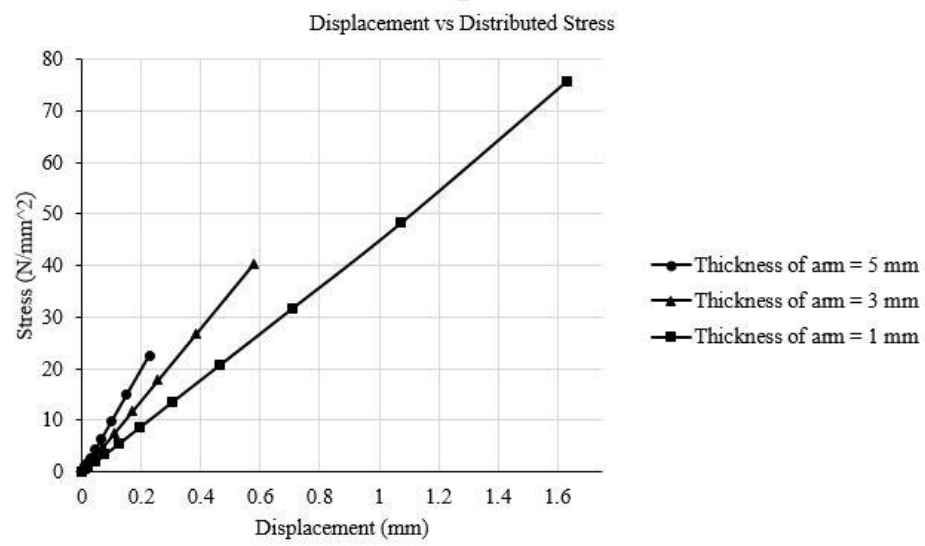

Figure 9. Displacement vs Distributed stress

\section{CONTROL ARCHITECTURE}

It is usually used to represent the ways in which the internally employed sensors and motors are deliberated to change robots' behavior. Five slave controllers and a master controller are needed to implement the actions needed to complete the tasks allocated to the robot. The precedence of one layer over the other would be discussed in the future paper work. Five different layers of the control architecture are discussed below. 


\subsection{When the robot is at semi or at open surface (Selective drive mechanism)}

When the robot is placed in the manhole, all the wheels of the robot need not to be powered to move inside the pipe. Since only the bottom two wheels from front and the bottom two wheels from the rear is in contact with the pipe surface. At that point it is necessary to cut off the power to the remaining wheels. This will help to reduce the power consumption. Same concept can be adopted when the robot is made to move outside the underground drainage system. This status would be updated to the microcontroller with the help of the ultrasonic sensors. Figure 10 shows the control architecture of selective drive mechanism.

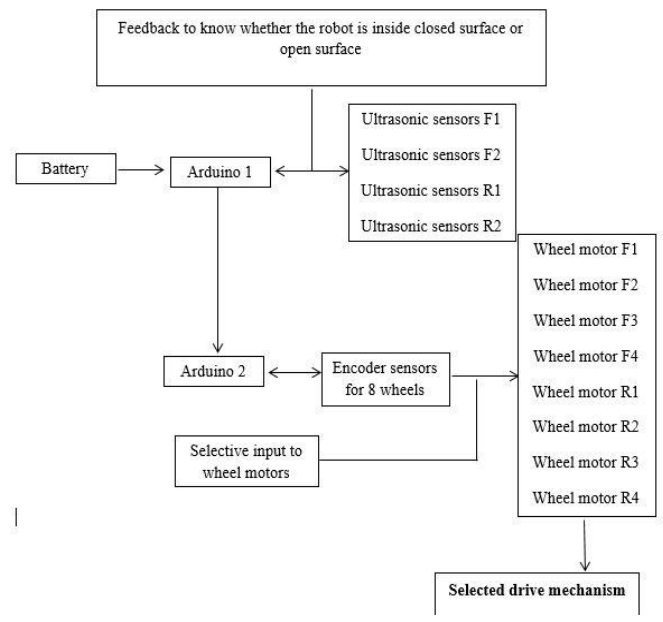

Figure 10. Selective drive mechanism

\subsection{Movement from manhole to closed pipe (Arm set mechanism)}

When the robot is entering the closed pipe from the manhole, the first set of wheels have to adapt itself to the pipe surface by getting the signal from ultrasonic sensor with the help of lead screw motor, in order to establish surface contact. Also at the same time the second set of wheels have to take the same set of signal by default for the first time. Otherwise the second set of wheel may face difficulty to enter in to the pipe, if they extend beyond the pipe diameter. One condition is that whenever the robot is at open surface the lead screw motor rotate to make the arm contract in order to make it move inside $200 \mathrm{~mm}$ pipe by default. Figure 11 shows the control architecture of arm set mechanism.

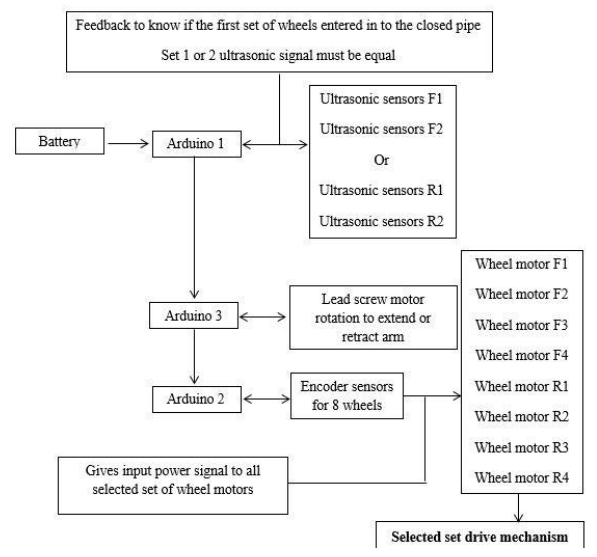

Figure 11. Arm set mechanism

\subsection{When the robot is inside the closed pipe (Full drive mechanism)}

When the robot is inside the pipe all the wheels have to be powered to get smooth motion. The condition is that any set of ultrasonic sensor signal should be equal. Otherwise the robot will assume that the 
robot is in open or semi open surface and will execute any of the above discussed conditions. Figure 12 shows the control architecture of full drive mechanism.

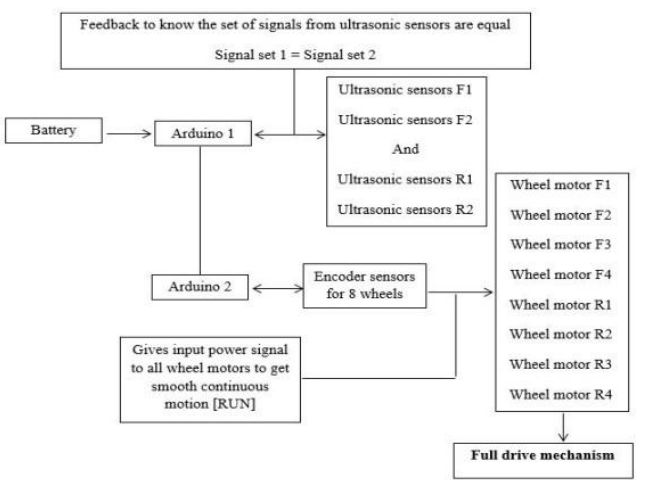

Figure 12. Full drive mechanism

\subsection{When the robot came across the clog in pipe (choke adjust mechanism)}

If the robot comes across the clog the IR optical proximity sensors will detect it and will send the signal to the controller which would send back a command to the wheel motors to stop the robot at a certain distance before the clog for the easy removal of the clog. And the lead screw motor have to rotate about a trivial amount to make the robot tightly hold with the surface. Figure 13 shows the control architecture of choke adjust mechanism.

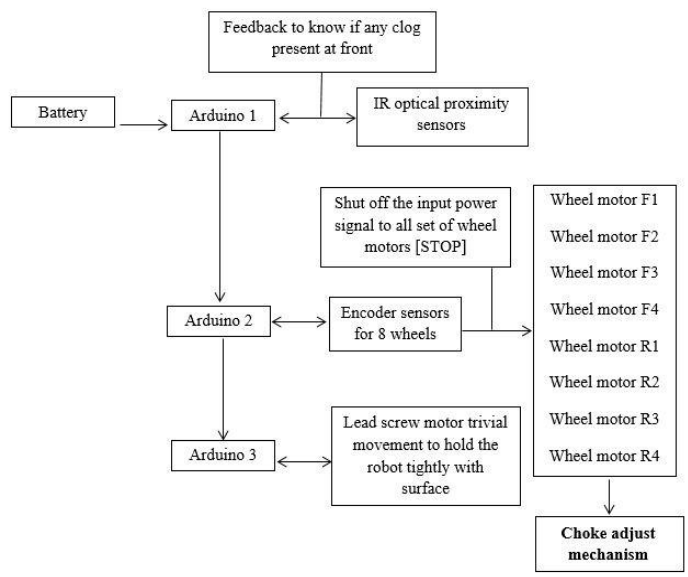

Figure13. Choke adjust mechanism

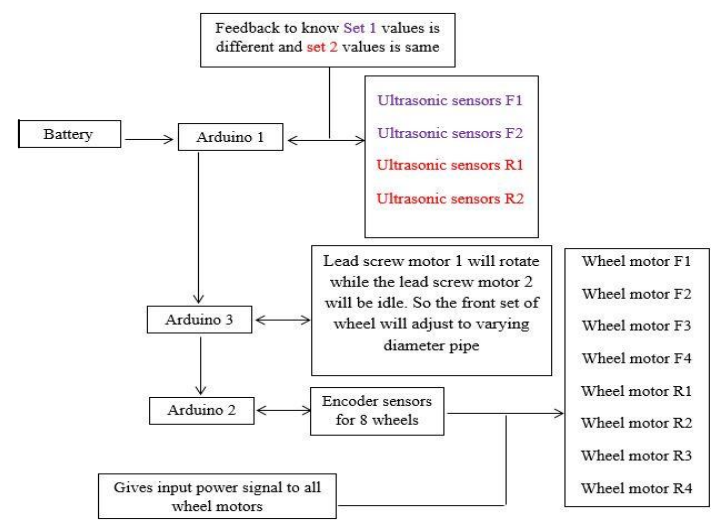

Figure 14. Small to big mechanism 


\subsection{When the robot moving from one pipe diameter to another pipe diameter (Small-to-Big mechanism)}

When moving from one pipe diameter to another diameter pipe, the one set of ultrasonic sensors at the end will show different value and the ultrasonic sensors set at the back will show same value. If the value of the set 1 ultrasonic sensor is different, the lead screw motor will rotate till the value of set 1 ultrasonic sensors become the same. And the same condition will be applicable for rear set. The reverse would be applicable for the robot to move from Big-to-Small diameter. Figure 14 shows the control architecture of Small-to-Big mechanism.

\section{FUZZY BASED CONTROL OF ROBOT}

Figure 14 shows the general fuzzy architecture. Fuzzy logic is a form of knowledge representation generally suitable for notions that cannot be defined precisely, but which depend upon their contexts. For our robot the fuzzy is employed in all the layers, for ex., to compare the input signal from the ultrasonic sensor in order to find out corresponding pipe diameter and the status of the work environment whether it is a closed, open or semi open environment. Figure 15 shows the fuzzy based architecture for the in pipe inspection robot. In the robot four ultrasonic sensor is employed. F1 is front sensor 1, F2 is front sensor 2, R1 is rear sensor 1 and R2 is rear sensor 2. Some of the rules used in the fuzzy logic are given below for a better understanding.

1. If Ultrasonic sensor F1 reading is in-between 7.5 to $10 \mathrm{~cm}$ and Ultrasonic sensor $\mathrm{F} 2$ reading is inbetween 7.5 to $10 \mathrm{~cm}$ and Ultrasonic sensor R1 reading is in-between 7.5 to $10 \mathrm{~cm}$ and Ultrasonic sensor R2 reading is in-between 7.5 to $10 \mathrm{~cm}$ then the pipe diameter in which the robot is moving is $20 \mathrm{~cm}$ and the work environment status is closed surface.

2. If Ultrasonic sensor F1 reading is in-between 7.5 to $10 \mathrm{~cm}$ and Ultrasonic sensor F2 reading is inbetween 10 to $12.5 \mathrm{~cm}$ and Ultrasonic sensor R1 reading is in-between 7.5 to $10 \mathrm{~cm}$ and Ultrasonic sensor $\mathrm{R} 2$ reading is in-between 7.5 to $10 \mathrm{~cm}$ then the pipe diameter in which the robot is moving is $20 \mathrm{~cm}$ and the work environment status is closed surface. But since the signal difference occurred in the front ultrasonic sensors it will be assumed that the robot is moving from 20 to $25 \mathrm{~cm}$ diameter pipe.

3. If Ultrasonic sensor F1 reading is in-between 12.5 to $15 \mathrm{~cm}$ and Ultrasonic sensor F2 reading is inbetween 12.5 to $15 \mathrm{~cm}$ and Ultrasonic sensor R1 reading is in-between 12.5 to $15 \mathrm{~cm}$ and Ultrasonic sensor $\mathrm{R} 2$ reading is in-between 12.5 to $15 \mathrm{~cm}$ then the pipe diameter in which the robot is moving is $30 \mathrm{~cm}$ and the work environment status is closed surface.

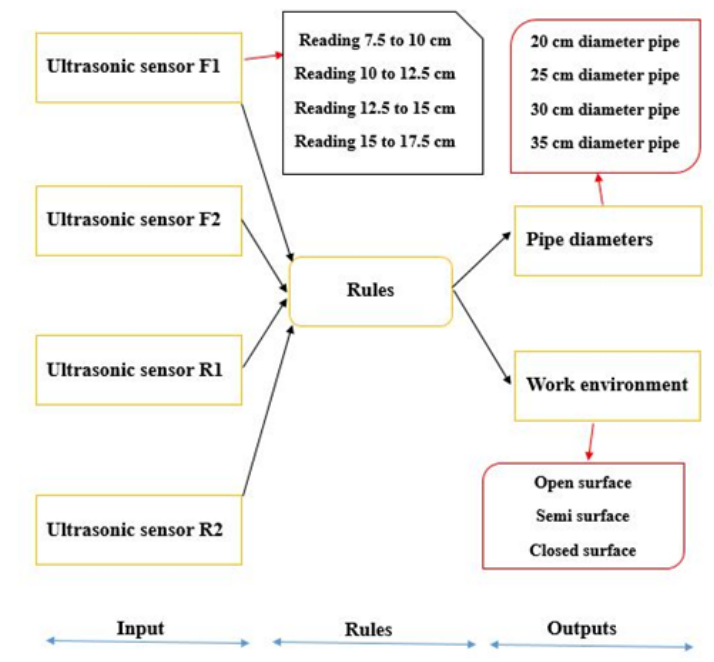

Figure 15. Fuzzy based architecture for the in pipe robot

\section{CONCLUSION}

The design parameters of the in-pipe robot are identified for prioritization and the relationship to the sub-parameters was established. Structural analysis reveals the best geometrical parameter i.e., the thickness of the links which would allow for better weight and payload capacity of the robot. The five layers of control 
architecture is also presented in detail. A fuzzy based control algorithm is proposed to arbitrate between the proposed five-layered control architecture for the purpose of controlling the robot towards achieving the objectives. The correlation of the parameters to the reconfigurability would be presented in the forthcoming papers from the authors.

\section{REFERENCES}

[1] Ajin M, Satheesh Kumar Gopal and Karthikeyan P , "Design, Structural and Kinematic Analysis of Reconfigurable Sewage Cleaning Robot", International Journal of Applied Mechanics and Materials, Accepted for publication, 2016.

[2] Nur Afiqah Binti Haji Yahya, Negin Ashrafi, Ali Hussein Humod, "Development and Adaptability of In-Pipe Inspection Robots", IOSR Journal of Mechanical and Civil Engineering, 11(4), pp. 1-8, 2014

[3] S. R. Jongerius, D. Lentink, "Structural Analysis of a Dragonfly Wing", International Journal of Experimental Mechanics, 50(9), pp. 1323-1334, 2010.

[4] Horodinca, Mihaita,Doroftei, Ioan,Mignon, Emmanuel,Preumont, André, A simple architecture for in-pipe inspection robots, Proceedings International Colloqium Mobile, 1-4, 2002.

[5] Atul Gargade, Dhanraj Tambuskar and Gajanan Thokal, Modeling and Analysis of Pipe Inspection Robot, International Journal of Emerging Technology and Advanced Engineering, 3(5), pp. 120-126, 2013.

[6] Leila Notash and Jingqiu Zhang, "Structural Synthesis of Kinematic Chains and Mechanisms", Integrated Design and Manufacturing in Mechanical Engineering, Springer Science \& Business Media Dordrecht Publishers, pp. 391398, 2002.

[7] D. Wang and C. B. Low, Modeling and Analysis of Skidding and Slipping in Wheeled Mobile Robots: Control Design Perspective, IEEE Transactions on Robotics, 24(3), pp. 676 - 687, 2008.

[8] D. Zlatanov, R. G. Fenton and B. Benhabib, Singularity analysis of mechanisms and robots via a motion-space model of the instantaneous kinematics, Proceedings of the 1994 IEEE International Conference on Robotics and Automation, 2, pp. 980-985, 1994.

[9] Satheesh G. Kumar, Nagarajan T and Srinivasa Y. G, Characterization of Reconfigurable Stewart Platform for Contour Generation, Robotics and Computer Integrated Manufacturing, 25, pp. 721-731, 2009.

[10] Satheesh G. Kumar, Nagarajan T and Singaperumal M , "Experimental Investigations on the Contour Generation of a Reconfigurable Stewart Platform", International Journal of Intelligent Mechatronics and Robotics, Volume 1, Number 4, pp. 87-99, 2011.

\section{BIOGRAPHIES OF AUTHORS}
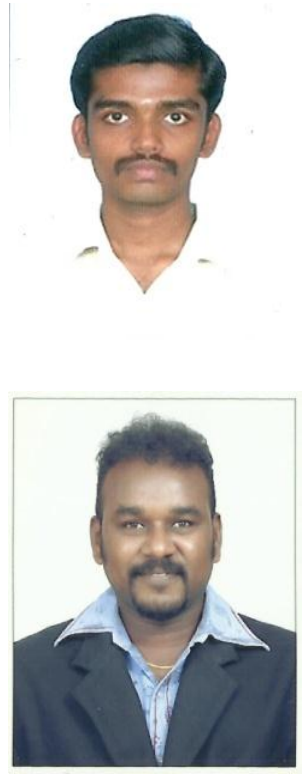

Ajin M isPost graduate scholar in Manufacturing Engineering at SSN College of Engineering, Kalavakkam (2016). He obtainedBachelor Degree in Mechanical Engineering from University College of Engineering, Nagercoil (India) in 2014.

His research interest are in Felds of Mobile robotics, Mechatronics, Kinematics of RoboticsNondistructive testing, CAD.

He has published two international journals and presented at two international conferences and has filed two patent. His current research include Mobile robot for inspection and cleaning in underground drainage system.

G. Satheesh Kumar received his M.E degree from REC Trichy, India in the year 2000 and his $\mathrm{PhD}$ degree from IIT Madras, India in the field of Parallel manipulators in the year 2008. He is currently working in SSN College of Engineering for the past two years and has worked for VEC, Chennai for three years earlier (2011-2014).

His research interest includes Robotics, Mechatronics, Automation, Kinematics and Dynamics. He has published over 30 journals and conference papers and has filed 5 patents in the field of robotics and automation.

He was working on developmental work of Virtual Remote Robotics Laboratory at IIT Madras (2009-2011) and an organizing member for the Indian National Robo Olympiad conducted for school children. He is actively involved in developing and testing prototypes of mobile robots and serial manipulators. His current research includes smart street lighting system, robot manipulators and robots for geriatric care. 